\title{
PICTORIAL COGNITIVE TASK RESOLUTION AND DYNAMICS OF EVENT-RELATED POTENTIALS
}

\author{
Josef Petrek
}

\author{
Department of Physiology, Faculty of Medicine and Dentistry, Palacky University, Olomouc, Czech Republic \\ e-mail:fesoj@tunw.upol.cz
}

Received: July 22, 2008; Accepted: September 17, 2008

Key words: Cognitive task/Personality traits/ERP

\begin{abstract}
Aims: To judge whether and how the character of the visual stimulus and type of cognitive task affects brain eventrelated potentials (ERPs).

Methods: ERPs to three types of visual stimuli (white blank oval on a dark background, unfolded cube and net of sixteen small squares) were recorded from nine scalp sites and saved on a computer. Special software was used for off-line analysis of the ERPs.

Results: The presentation of each of the three visual stimuli used was followed by ERPs consisting of two negative (N160, N340) and one positive (P220) components. The character of the stimulus did not affect the latency of ERPs components. However, the type of visual stimuli affected the amplitude. The most conspicuous changes were shown by the N340 ERPs component. Its average amplitude in comparison with reference amplitude was always significantly higher during the first cognitive task ("Choose the cube that can be folded up from the unfolded cube!") and significantly lower than reference amplitude during the second cognitive task ("Complete the missing part of a figure with the appropriate item!"). It was also shown that subjective personality traits such as nervousness, spontaneous aggressivity and emotional lability had an influence on the recovery phase of the experiment affecting the average amplitude of N340
\end{abstract}

Conclusion: The results revealed that the cognitive processes underlying successful resolution of two pictorial cognitive tasks affected differently the activity of systems giving rise to visual ERPs.

\section{INTRODUCTION}

Event-related potentials (ERPs) are currently considered a good tool for the study of different aspects of language processing and comprehension (see e.g. ${ }^{16,37,41}$ ). Their analysis also contributes significantly to knowledge of neural processes that underlie a highly specific skill in

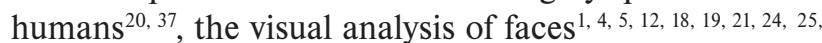

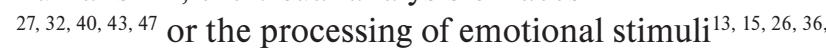
$28,44,45$. Therefore, it is not surprising that the recording and analysis of ERPs affect all areas of cognitive neurosciences.

In our recent study ${ }^{38}$ we used the ERP technique to analyze the impact of an instant cognitive activity (selection of two identical pictures in a group of similar pictures) on the behaviour of ERPs to visual stimuli (drawing of living creatures or common objects). The dynamics of the spontaneous EEG was also observed ${ }^{39}$. It was shown that the cognitive processes underlying successful selection of two identical pictures from a group of similar ones affected both the amplitude N160 and N340 ERPs components and the characteristics of the event-related desynchronization.

Taking into account the findings we decided to carry out an experiment with the aim of judging whether and how the character of the visual stimulus and the type of cognitive task can affect brain potentials or activity of other functional systems. Therefore, special attention was paid to: (a) the dynamics of ERPs components, (b) the character of an ongoing EEG activity and (c) some autonomic (heart rate) and metabolic parameters (minute ventilation, oxygen consumption and carbon dioxide production).

Answer to these questions will be given in several presentations. This project seeks an answer to the first question.

\section{METHODS}

\section{Subjects}

Fifteen right-handed female students from the Medical Faculty of Palacký University in Olomouc participated in the experiment. Records of only 12 were analysed - one female stopped the experiment at her own request and two subjects were discarded because of a high artefact rate. All students were twenty-one-year old and they reported taking no medication and had no history of neurological, opthalmological or systemic disease. Their vision was normal or corrected to normal. Written informed consent was obtained from all participants prior to the start of the experiment.

\section{Stimuli}

Three types of $3500 \mathrm{~ms}$ long visual stimuli were used to test electrophysiological correlates of participants' cogni- 
tive activity. The first type of stimuli (white blank oval on a dark background) constituted the reference stimuli and their presentation did not require any cognitive activity on the part of the subject. The other two types (unfolded cube and the net of sixteen small squares) represented the test stimuli the presentation of which was followed by specific cognitive and motor activity. The test stimuli appeared in fifteen variants of which seven formed the unfolded cube (left half of Figure 1) and the remaining eight on the net of squares (right half of Figure 1). The graphic images for all variants of test stimuli were taken from the book of Carter and Russell ${ }^{7}$.

A balanced $15 \times 15$ Latin square design was used to arrange blocks of test stimuli into an experimental sequence. The fifteen experimental sequences were created altogether - one sequence for each of participant. Each of the fifteen sequences contained fifteen blocks of test stimuli (unfolded cube seven times, net of squares eight times), fifteen blocks of reference stimuli (white blank oval on a dark background) and fifteen warning stimuli (five asterisks in a white oval on black background) preceding each block of stimuli. The number of stimuli in blocks was as follows: each test block contained 160 stimuli, each reference block only 10 stimuli.

Besides fifteen experimental sequences, one practice sequence was created. However, this contained only one block of reference visual stimuli and one block of 100 test stimuli (cube and net of squares - fifty times each), which did not belong to any of experimental sequences.

\section{Presentation of stimuli}

SciWorks Version 5, Experimenter's WorkBench 32 Data Acquisition and Experiment Control Software (DataWave Technologies, Longmont, CO) was used to control stimulus presentation, regulate the timing of events, and record the responses of participants. A photodiode was used to record the moment of a visual stimulus appearing on the screen. Each of the fifteen volunteers was tested individually in a darkened, sound-attenuated, and electrically shielded room. During the experiment, the participant was seated in a comfortable chair approximately $120 \mathrm{~cm}$ in front of the computer screen (SONY, Model: SDM-X72). Sequences of visual stimuli were presented on a computer screen with resolution of $1024 \times$ 768 pixels. A second monitor, located outside the testing room, allowed the experimenter to view the stimulus presented on each trial.

\section{Procedure}

The experiment started with the presentation of the practice sequence. After its completion the experimental sequence presentation was begun. At first $3500 \mathrm{~ms}$ long warning stimulus appeared on the screen. After 500 ms after its termination, the first picture of a reference block stimuli appeared on the screen. Other pictures (10 in sum) followed the first one with inter-stimulus interval of $500 \mathrm{~ms}$. In $500 \mathrm{~ms}$ after the tenth reference stimulus ending ensued further warning stimulus. It signalled the beginning of the presentation of the test block stimuli. The presentation of them (160 in sum) abided by the

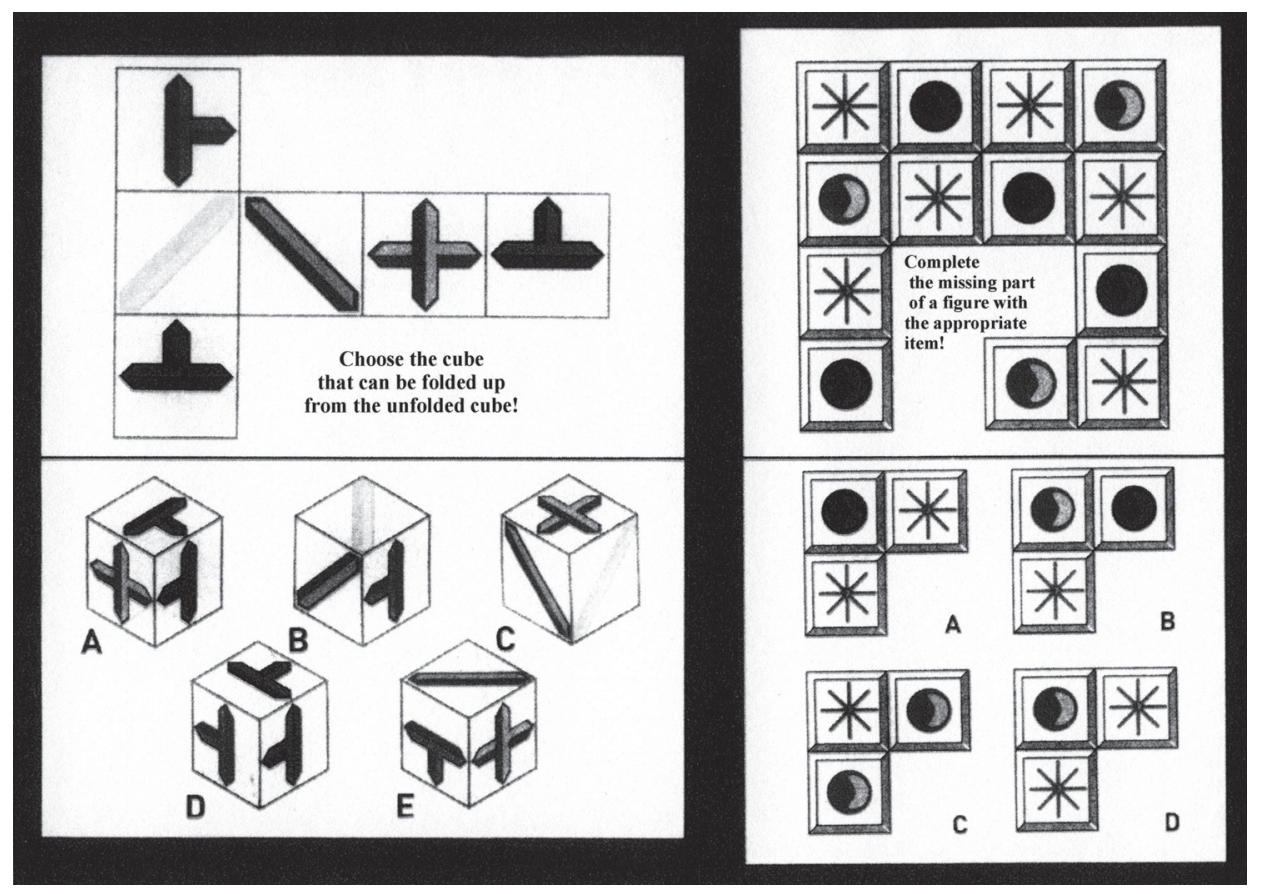

Fig. 1. Example of the two types of test visual stimuli used in the experiment to initiate a prearranged cognitive activity - "unfolded cube" signalized the choice of the cube that can be folded up from the unfolded cube, "net of sixteen small squares" the completion of missing part of the figure.

Graphic images for all variants of test stimuli were taken from the Czech translation of a book by Carter and Russell (2002) and modified. Coloured pictures were presented. 

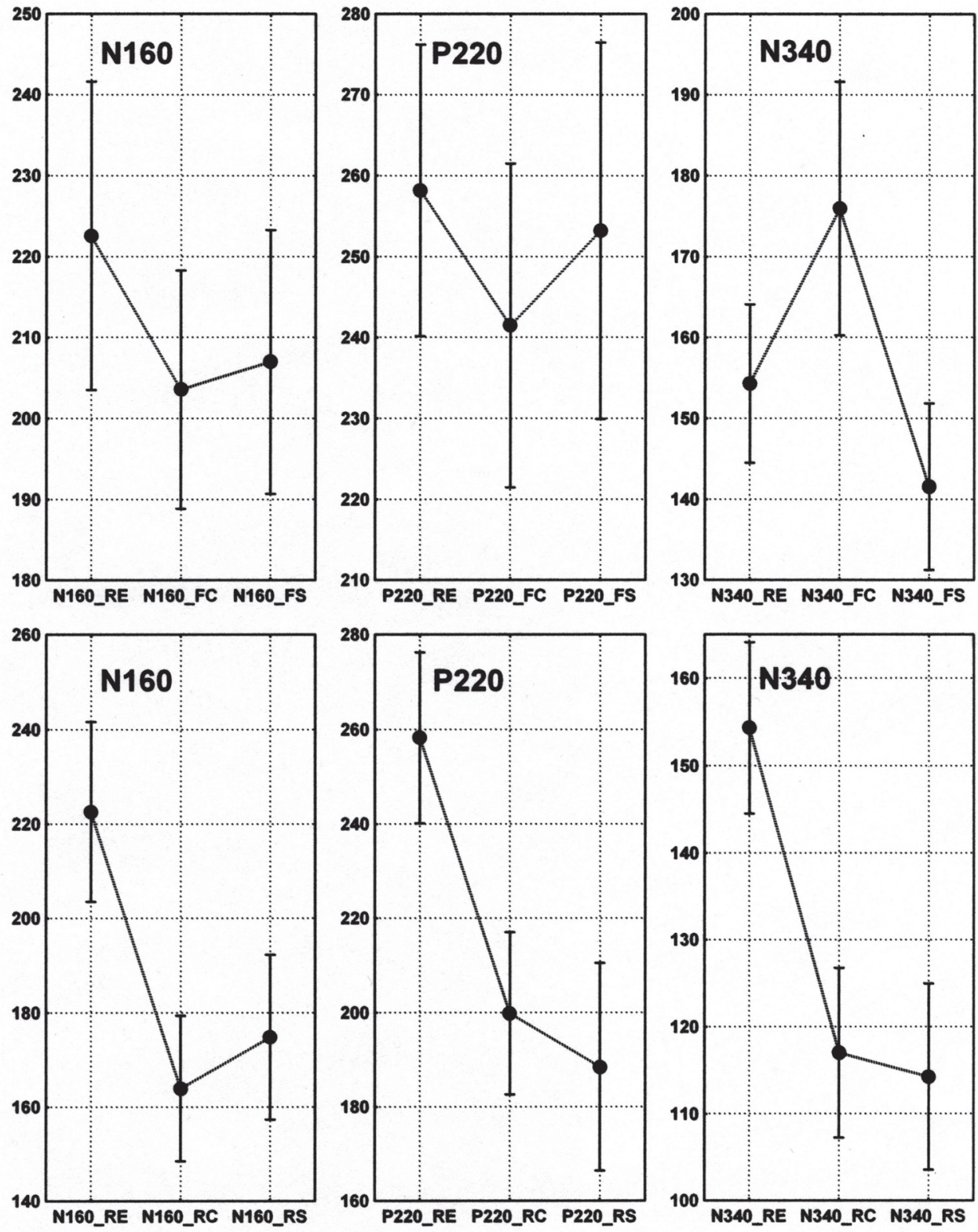

Fig. 2. Average amplitudes of N160, P220 and N340 (in AD/mV) to reference stimuli (_RE), to "unfolded cubes" (_FC/_RC) and to "nets of sixteen small squares" (_FS/_RS) during the cognitive task solving period (upper half) and during the rest period (lower half).

Calibration: $40 \mathrm{AD} / \mathrm{mV}=1 \mu \mathrm{V}$; Black circles - average values; Vertical bars - 0.95 per cent confidence intervals.

same rules as the presentation stimuli of the reference block. The last stimulus of the test block was followed by a forty-second pause without any visual stimuli.

After pause termination, the warning stimulus appeared on the screen again and the described procedure was repeated once more with the next available blocks of reference and test stimuli. The whole experiment lasted approximately two hours.

Before the experiment began, subjects were instructed to restrain from blinking, moving their eyes and swallowing during the presentation of the stimuli. They were also asked to focus on asterisks (warning stimulus) whenever they appear on the screen. Concurrently they were told to modulate their next behaviour according to the character of the visual stimulus that replaced the warning stimulus.

When the warning stimulus replaced the white blank oval on black background (reference stimulus), the subjects merely viewed the stimulus appearing on the screen. On the other hand, when the warning stimulus replaced the test stimulus of whichever block (unfolded cube or net of sixteen small squares), the subjects had to solve the preassigned cognitive task. This means that subjects had to choose which cube from the offer can be folded up from the presented net of a cube (the first cognitive task) or they had to choose the satisfactory item from 

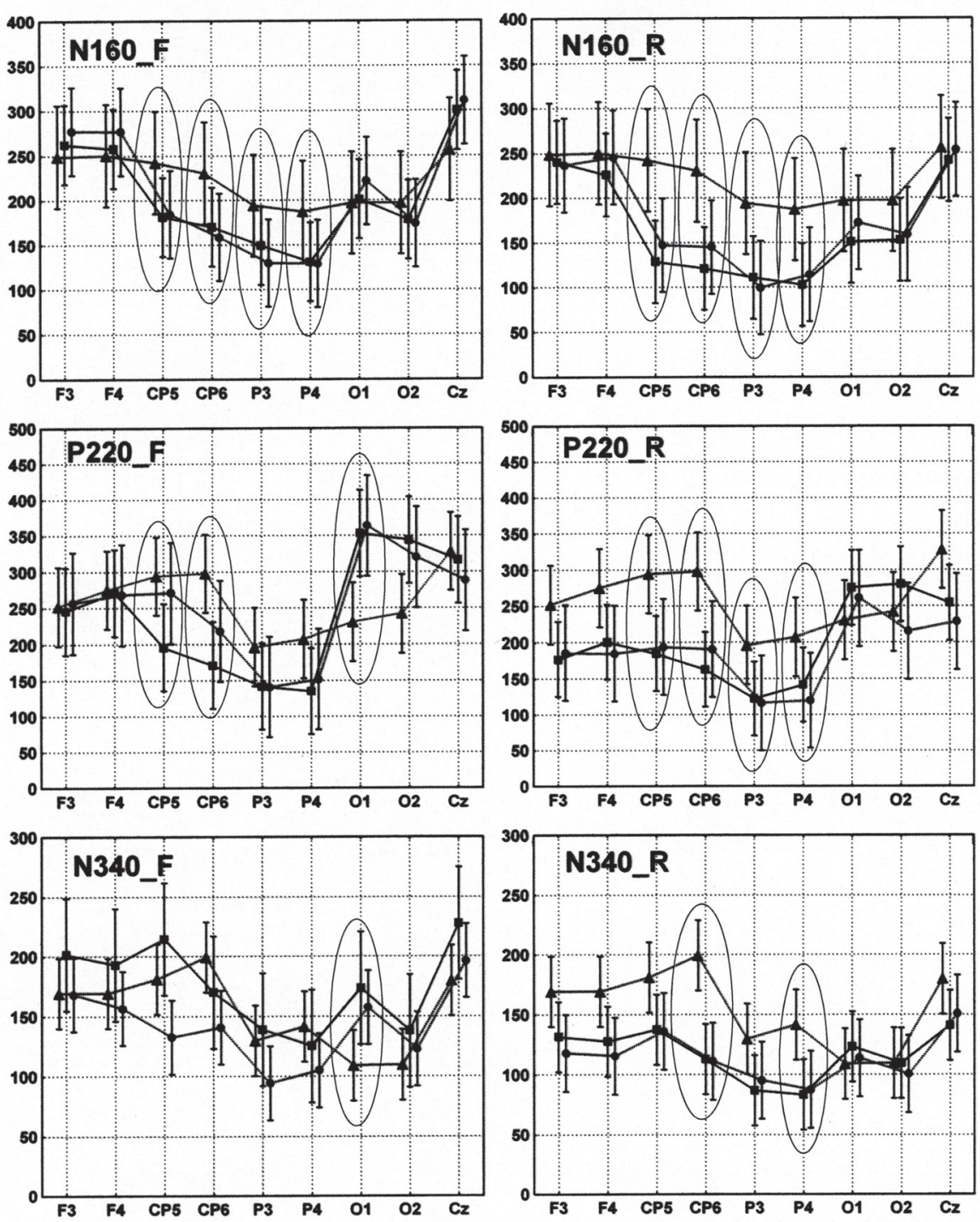

Fig. 3. Average amplitudes of $\mathrm{N} 160, \mathrm{P} 220$ and $\mathrm{N} 340$ (in $\mathrm{AD} / \mathrm{mV}$ ) to reference stimuli (triangles), to unfolded cubes (squares) and to nets of sixteen small squares (black circles) during the cognitive task solving period (left half, _F) and during the rest period (right half, _R) in different scalp recording sites (abscissa).

Significant differences between averages $(p<0.05$ ] are encircled. Calibration: $40 \mathrm{AD} / \mathrm{mV}=1 \mu \mathrm{V}$; Vertical bars -0.95 per cent confidence intervals.

the offer to replenish the missing part of the figure (the second cognitive task).

The result of task solving was announced aloud to the experimenter and simultaneously a mouse button was pressed to measure the reaction time. If their choice was correct, the experimenter told them and at the same time asked them to view only the stimuli appearing on the screen without any cognitive activity. If their choice was not correct, they were asked to continue task solving until they were successful.

\section{Data acquisition}

EEG was recorded from 9 scalp sites by tin electrodes attached to an elastic cap (Electro-Cap International, Eaton, $\mathrm{OH}$ ). The electrodes were placed in scalp positions $\mathrm{F} 3 / 4, \mathrm{CP} 5 / 6$ (a crossing point between T3/4-P3/4 and C3/4-T5/6), P3/4, O1/2 and Cz. One electrode was also placed at the outer canthus of the right eye to monitor eye movements. Scalp electrodes were referred to linked earlobes. The ground electrode was placed at Fpz. Electrodes impedance was kept below $3 \mathrm{k} \Omega$ throughout the experiment.

The EEG signal was amplified by the cascade of two amplifiers (amplifiers EKe, OTE Biomedica, Firenze, and Amplifier Neuralynx, Tucson, Arizona) with bandpass of $0.01-100 \mathrm{~Hz}$ and continuously sampled at $256 \mathrm{~Hz}$ per channel by an A/D converter; the resolution of the system is $0.122 \mu \mathrm{V} / \mathrm{bit}$. The digitised records were saved on the hard disk of a computer for later off-line replay and analysis.

After the end of the experiment all participants were administered the Freiburg Personality Inventory (FPI) 
$\left(\right.$ ref..$^{30}$ ) in the modified version for Czech and Slovak populations.

\section{Data analysis}

SciWorks Version 5, DataWave CP Analysis Module and Data Editing Software were used for off-line analysis of EEG records. The analysis ran as follows: A visual elimination of distorted records, digital filtering of records (bandpass 1-30 Hz), cutting filtered records for averaging (the start points of the cut segment of EEG records were time-locked to photodiode signal) and detection of ERPs by averaging. Then maximal positive and negative amplitudes from baseline, calculated by software using the first five points of the waveform, were measured in time windows 120-250 ms (N160 and P220) and 250-400 ms (N340 ERPs component). StatSoft software package (StatSoft, Tulsa, OK) was used for statistic processing of the ERPs data.

\section{RESULTS}

The presentation of each of the three visual stimuli used in our experiment (white blank oval on a dark background, unfolded cube or net of sixteen small squares) was followed by an event-related potential (ERP) consisting of two negative (N160, N340) and one positive (P220) components. The character of the stimulus did not affect the latency of ERPs components but the latency was affected by the site of scalp recording electrode - the latencies were significantly shorter in occipital and parietal scalp sites than in frontal ones. However, the type of visual stimuli or rather the mental activity related to them markedly affected the amplitude of all ERPs components.

\section{Functional state of the brain and ERPs amplitude dynamics}

The average amplitudes of all visual ERPs components registered during the solution of the first ${ }^{\mathrm{a}}$ and the second $\mathrm{d}^{\mathrm{b}}$ cognitive task (upper half of the Figure 2) and also in the rest period ${ }^{c}$ (bottom half of the Figure 2) differed from the average amplitudes of ERPs to reference stimuli. The ERPs component N340 illustrates this explicitly (see the third column in Figure 2). In comparison with the reference amplitude (N340_RE), its amplitude was significantly higher during the solution of the first cognitive task (N340_FC) than during the solution of the second one (N340_FS). On the other hand, in the rest period, the average N340 amplitudes to both types of test stimuli were significantly lower than the reference amplitude and they did not show any significant differences.

The average amplitudes of $\mathrm{N} 160$ and P220 components recorded in our subjects during the mental task solving and in the rest period behaved similarly as N340

\footnotetext{
a "Choose the cube that can be folded up from the unfolded cube!"

b "Complete the missing part of the figure with the appropriate item!"

c The period following successful mental task resolution in which presentation of visual stimuli goes on.
}

amplitudes in the rest period. In contrast to N340, however, the average amplitudes measured during the mental task solving (left and middle graphs in upper half of Figure 2) did not reach statistical significance compared with the corresponding reference amplitude. The high variability of N160 and P220 could be the most probable cause. In the rest period (bottom half of Figure 2) there were no differences between the amplitude behaviour of N340 and the other two ERPs components.

The average ERPs amplitude is affected not only by the type of a cognitive task but also by the site of the scalp-recording electrode (see Figure 3). The post hoc comparison of averages (Duncan's test) showed that the reference stimuli (triangles in Figure 3) produced ERPs with significantly lower amplitude in sites P3, P4, O1 and $\mathrm{O} 2$ than in the rest scalp areas. The amplitudes of ERPs to test stimuli in principle showed similar dynamics though some differences appeared (see later). Interesting is also the finding that the momentary functional states of the subject's nervous system (solving cognitive tasks or only a passive viewing of visual stimuli without any cognitive activity) did not affect the average amplitudes of individual ERPs components to test stimuli (compare the black squares and dots in Figure 3).

A detailed inspection of the two graphs in the upper line in Figure 3 shows that regardless of the functional state of the subject's brain both test stimuli produced N160 ERPs component in sites CP5, CP6, P3 and P4 with significantly lower amplitude than did the reference stimuli. Similarly behaved also the average amplitude of P220 registered during the rest period (see the middle graph in the right column in Figure 3) and with a certain reservation also the average amplitude of N340 - the letter shows significant differences only at CP6 and P4 recording sites (see the bottom graph in the right column in Figure 3).

The average amplitudes of ERPs component P220 and N340 measured during the mental task solving showed a slightly different picture of dependence on the site of recording electrode. Unlike the N160 ERPs component, they reached the highest average amplitude at the $\mathrm{O} 1$ recording site during the cognitive task (see the middle and bottom graphs in Figure 3). Further, the amplitudes of P220 and 340 ERPs components showed other difference. The amplitude of both ERPs components at parietal recording sites (CP5 and CP6) depended on the type of the stimulus that elicited them. The average P220 amplitude was significantly lower than the reference amplitude only when the first type of test stimuli (unfolded cube) was presented. The amplitude of N340 showed the same dynamics only if the second type of test stimuli (net of squares) appeared.

\section{Personality traits and amplitude ERPS components}

Simple Linear Correlation analysis showed that some subject's personality traits (nervousness - FPI1, spontaneous aggressiveness - FPI2, and emotional lability - N) affected the average amplitude of N340 ERPs component in the recovery phase of the experiment. Conversely, the 
score of FPI2 only did so during the cognitive task resolution period. Amplitudes of the N160 and P220 ERPs components showed no dependence on personality traits of our subjects.

The average amplitude of the N340 ERPs component expressed as percentage of the reference amplitude was the higher, the lower the score each of three FPI parameters was. This means that the average N340 amplitude reached the smallest values in subjects showing an increased excitability of the nervous system (high score of FPI1 and N) and high readiness to admit personal weaknesses, passions and particularly tendencies to aggression (FPI2)

\section{DISCUSSION}

The results show that the appearance of the visual stimulus on the screen produced in all our subjects, in each scalp electrode, an event-related potential (ERP) with two negative (N160 and N340) and one positive (P220) components. In principle, the type of the stimulus (white blank oval on a dark background, unfolded cube or net of sixteen small squares) did not affect the latency of ERPs components but the site of scalp recording electrode did - latencies were slightly shorter in parietal and occipital brain areas. The latencies of visual ERPs analyzed in our earlier paper ${ }^{38}$ showed similar dynamics. In contrast to latencies, however, the type of visual stimulus or rather the mental activity related to it, markedly affected the amplitude of all ERPs components.

We also found that cognitive processes underlying the successful solution of two cognitive tasks affect differently the activity of systems giving rise to visual ERPs. In particular, the amplitude changes of the negative component N340 demonstrated it graphically. The average amplitudes of N340 compared to the reference amplitude were always significantly higher during the first cognitive task solving ("Choose the cube that can be folded up from the unfolded cube!") than during the second cognitive task solution ("Complete the missing part of a figure with an appropriate item!”).

Why the amplitude N340 behaved as it did, we are unsure. However, some literature data might help us to understand its behaviour.

Barrett and Rugg $^{3}$ in an experiment using a relatedness judgment task (subjects determined whether pairs of sequentially presented pictures were semantically associated) observed that a negative ERPs component N300 is sensitive to semantic manipulation - the amplitude of N300 was more negative to non-associated than to associated pictures. McPherson and Holcomb ${ }^{33}$ obtained similar results. They demonstrated that in a relatedness judgment task using pictures, the frontally distributed N300 was larger in unrelated than in related pictures.

The results of West et al. ${ }^{48}$ are not in conflict with those listed above. In special experiments (subjects viewed congruous and incongruous picture stories) they showed that the amplitude of the negative ERPs component peak- ing at $\sim 325 \mathrm{~ms}$ after stimulus onset is sensitive to the global coherence of the final picture with a prior context. A final picture that was incongruous with the context established in the preceding series of pictures elicited a more negative waveform than the final picture that was congruous with the preceding context. According to Kounios ${ }^{31}$, West and Holcomb's results suggest that N300 reflects a mechanism specialized in processing pictorial or imagistic representations, i.e. it could plausibly reflect the initial activation of the pictorial or imagistic discourse-level semantic representation.

According to Hamm et al. ${ }^{22}$, the N300 is sensitive to categorical-level mismatches (in their experiments it was elicited only when the mismatch was between categories but not when it was within a category). Thus the N300 probably reflects early semantic categorization of an object, which occurs prior to the determination of more specific identity information. Eddy et al. ${ }^{17}$ also state that the anterior negativity (N300) reflects processing of object-specific representations.

For completeness, the findings of Carretié et al. ${ }^{9-11}$ showing that N300 is also an emotion-sensitive ERPs component are relevant. Its amplitude is greater in response to activating positive visual stimuli at frontal sites and for negative ones at parietal sites. The results of Rossignol et al. ${ }^{42}$ - N300 is enhanced in amplitude for fearful stimuli, and Schutter el al. ${ }^{46}$ - N300 is more negative for the angry facial expression, are not in conflict with previous findings, either.

The literature shows that that N300 in the exclusively pictorial task is specific to the semantic processing of pictures and is not due to linguistic mediation. The N300 is sensitive to semantic rather than physical properties of the picture identification task. It may reflect the early semantic categorization of object stimuli, which precedes definitive identification. Naturally, all of this takes place against a given emotional background which undoubtedly affects the momentary activity of the nervous system.

Our data corroborate the latter statement. The analysis revealed that subjects showing increased excitability of the nervous system (a high score of nervousness - FPI1, and emotional lability - $\mathrm{N}$, and readiness to admit personal weaknesses, passions and particularly tendencies to aggression - FPI2) had lower average N340 amplitude.

From the literature, it can be inferred that the greater amplitude of N340 in our experiments during the first cognitive task solving indicates increased activity of brain systems taking part in semantic categorization of the presented visual stimuli. Successful resolution of the first cognitive task was more demanding mentally than the second one.

Our results are not in conflict with this assumption. We demonstrated that mere passive viewing of both visual stimuli (an unfolded cube and a net of small squares) without any cognitive activity (the rest period of our experiment) is followed by ERPs amplitudes of N340 showing no differences. Indirect evidence for our assumption is also provided by the results of Conley et al. ${ }^{14}$, demonstrating that amplitude of slow negative shift (N300-600) 
increases linearly with increasing mental load. The same applies Zhou et al. ${ }^{49}$, who showed that, compared to addition and subtraction, multiplication elicited a greater N300 from the frontal electrodes.

The share of frontal and parietal brain areas in the processing of visual information during the cognitive task solving has been repeatedly demonstrated (see e.g. $2,6,8,23,29,35)$. The presented findings as well as our earlier results are in agreement. However, only further experiments will increase understanding of the behaviour of electrophysiological correlates related to activity of brain systems participating in pictorial cognitive task solving.

\section{REFERENCES}

1. Allison T, Puce A, Spencer DD, McCarthy G. Electrophysiological studies of human face perception. I: Potentials generated in occipitotemporal cortex by face and non-face stimuli. Cereb Cortex 1999; 9:415-30.

2. Bar M. A cortical mechanism for triggering top-down facilitation in visual object recognition. J Cogn Neurosci 2003; 15:606-9.

3. Barrett SE, Rugg MD. Event-related potentials and the semantic matching of pictures. Brain Cogn 1990; 14:201-12.

4. Bentin S, Allison T, Puce A, Perez E, McCarthy G. Electrophysiological studies of face perception in humans. J Cogn Neurosci 1996; 8:551-6.

5. Bentin S, Golland Y, Flevaris A, Robertson LC, Moscovitch M. Processing the trees and the forest during initial stages of face perception: Electrophysiological evidence. J Cogn Neurosci 2006 18:1406-21.

6. Beteleva TG, Farber DA. Role of the frontal cortical areas in the analysis of visual stimuli at conscious and unconscious levels. Human Physiol 2002; 28:511-9.

7. Carter P, Russel K. Workout for a balanced brain. Quatro Publishing:London, 2001; Czech translation: Efektivni vyuziti mozku. Rebo Productions CZ: Dobrejovice, 2002.

8. Carretié L. Neural response to sustained affective visual stimulation using an indirect task. Exp Brain Res 2006; 174:630-7.

9. Carretié L, Inglesias J. An ERP study on the specificity of facial expression processing. Int. J Psychophysiol 1995; 19:183-92.

10. Carretié L, Inglesias J, García T, Ballesteros M. N300, P300 and the emotional processing of visual stimuli. Electroenceph clin Neurophysiol 1996; 103:298-303.

11. Carretié L, Inglesias J, García T. A study on the emotional processing of visual stimuli through event-related potentials. Brain Cogn 1997; 34:207-17.

12. Cassia VM, Kuefner D, Westerlund A, Nelson CA. Modulation of face-sensitive event-related potentials by canonical and distorted human faces: The role of vertical symmetry and up-down featural arrangement. J Cogn Neurosci 2006; 18:1343-58.

13. Codispoti M, Ferrari V, Bradley MM. Repetitive picture processing: Autonomic and cortical correlates. Brain Res 2006; 1068:213-20.

14. Conley E, Michalewski A, Starr A. Scanning auditory short-term memory is associated with systematic changes of sensory (N100) and cognitive (N300-600) components of event related brain potentials. Electroenceph clin Neurophysiol 1997; 103:24.

15. Cuthbert BN, Schupp HT, Bradley MM, Birbaumer N, Lang PJ Brain potentials in affective picture processing: covariation with autonomic arousal and affective report. Biol Psychol 2000; 52:95111.

16. Druks J. Verbs and nouns - a review of the literature. J Neuroling 2002; 15:289-315.

17. Eddy M, Schmid A, Holcomb PJ. Masked repetition priming and event-related brain potentials: A new approach for tracking the time-course of object perception. Psychophysiol 2006; 43:564-8.

18. Eimer M. The face-specific N170 component reflects late stages in the structural encoding of faces. Neuroreport 2000; 11:2319-24.
19. Eimer M, McCarthy, Rosaleen A. Prosopagnosia and structural encoding of faces: Evidence from event-related potentials. Neuroreport 1999; 10:255-9.

20. Gunter TC, Bach P. Communicating hands: ERPs elicited by meaningful symbolic hand postures. Neurci Letters 2004; 372:52-6.

21. Goffaux V, Gauthier I, Rossion B. Spatial scale contribution to early visual differences between face and object processing. Cogn Brain Res 2003; 16:416-24.

22. Hamm JP, Johnson BW, Kirk IJ. Comparison of the N300 and N400 ERPs to picture stimuli in congruent and incongruent contexts. Clin Neurophysiol 2002; 113:1339-50.

23. Henson RN, Goshen-Gottstein Y, Ganel T, Otten LJ, Quayle A, Rugg MD. Electrophysiological and haemodynamic correlates of face perception, recognition and priming. Cereb Cortex 2003; 13:793-805

24. Herrmann MJ, Aranda D, Ellgring H, Mueller TJ, Strik WK, Heidrich A, Fallgatter AJ. Face-specific event-related potential in humans in independent from facial expression. Int J Psychophysiol 2002; 45:241-4.

25. Herrmann MJ, Ehlis A-C, Ellgring H, Fallgater AJ. Early stages (P100) of face perception in humans as measured with event-related potentials (ERPs). J Neural Transm 2005; 112:1073-81.

26. Hot P, Saito Y Mandai O, Kobayashi T, Sequiera H. An ERP investigation of emotional processing in European and Japanese individuals. Brain Res 2006; 1122:171-8.

27. Itier J, Taylor MJ: N170 or N1? Spatiotemporal differences between object and face processing using ERPs. Cereb Cortex 2004; 14:13242.

28. Junghöfer M, Bradley MM, Elbert TR, Lang PJ. Fleeting images: A new look at early emotion discrimination. Psychophysiol 2001; 38:175-8.

29. Kawasaki H, Adolphs R, Kaufman O, Damasio H,Damasio AR, Granner M, Bakken H, Hori T, Howard MA. Single-neuron responses to emotional visual stimuli recorded in human ventral prefrontal cortex. Nat Neurosci 2001; 4:15-6.

30. Kollarik T, Poliakova E, Ritomsky A. Freiburský osobnostný dotaznik (in Slovak). Bratislava:Psychodiagnostické a didaktické testy, 1984.

31. Kounios J. A neural mechanism for non-verbal discourse comprehension. Trends Cogn Sci 2002; 6:272-75.

32. McCarthy G, Puce A, Belger A, Allison T. Electrophysiological studies of human face perception: II. Response properties of facespecific potentials generated in occipitotemporal cortex. Cereb Cortex 1999; 9:431-44.

33. McPherson WB, Holcomb PJ. An electrophysiological investigation of semantic priming with pictures of real objects. Psychophysiol 1999; 36:53-65.

34. Neville HJ, Coffey SA, Lawson DS, Fischer A, Emmorey K, Bellugi U. Neural systems mediating American Sign Language: Effect of sensory experience and age of acquisition. Brain Lang 1997; 57:285-308.

35. Northoff G, Richter A, Gessner M, Schlagenhauf F, Fell J, Baumgart F, Kaulisch T, Kötter R, Stephan K, Leschinger A, Hagner T, Bargel B, Witzel T, Hinrichs H, Bogerts B, Scheich H, Heinze HJ. Functional dissociation between medial and lateral prefrontal cortical spatiotemporal activation in negative and positive emotions: A combined fMRI/MEG study. Cereb Cortex 2000; 10:93-107.

36. Polomba D, Angrill A, Mini A. Visual evoked potentials, heart rate responses and memory to emotional pictorial stimuli. Int $\mathbf{J}$ Psychophysiol 1997; 27:55-67.

37. Petrek J. Brain activity during language processing (in Czech). Cs Fyziol 2004; 53:125-31.

38. Petrek J. Selection of two identical pictures from a group of similar ones: I. Dynamics of event-related potentials. Activ Nerv Sup 2007; 49:93-100.

39. Petrek J. Selection of two identical pictures from a group of similar ones: II. Changes in ongoing EEG activity. Activ Nerv Sup 2008; 50:3-8.

40. Puce A, Allison T, McCarthy G. Electrophysiological studies of human face perception. III: Effects of top-down processing on facespecific potentials. Cereb Cortex 1999; 9:445-58. 
41. Pulvermüller F. Words in the brain's language. Behav Brain Sci 1999; 22:253-336.

42. Rossignol M, Philippot P, Douilliez C, Crommelinck M, Campanella $\mathrm{S}$. The perception of fearful and happy facial expression is modulated by anxiety: an event-related potential study. Neurosci Letters 2005; 377:115-120.

43. Rossion B, Gauthier I, Tarr MJ, Despland P, Bruyer R, Linnote $\mathrm{S}$, Grommelinck M. The N170 occipito-temporal component is delayed and enhanced to inverted faces but not to inverted objects: An electrophysiological account of face-specific processes in the human brain. Neuroreport 2000; 11:69-72.

44. Schupp HT, Junghöfer M, Weike AI, Hamm AO. Emotional facilitation of sensory processing in the visual cortex. Psychol Sci 2003; 14:7-13.
45. Schupp HT, Junghöfer M, Weike AI, Hamm AO. The selective processing of briefly presented affective pictures: An ERP analysis. Psychophysiol 2004; 41:441-9.

46. Schutter DJLG, de Haan EHF, van Honk J. Functionally dissociated aspects in anterior and posterior electrocortical processing of facial threat. Int J Psychophysiol 2004; 53:29-36.

47. Taylor MJ, Edmonds GE, McCarthy G, Allison T. Eyes first! Eye processing develops before face processing in children. Neuroreport 2001; 12:1671-6.

48. West WC, Holcomb PJ. Event-related potentials during discourselevel semantic integration of complex pictures. Cogn Brain Res 2002; 13:363-75.

49. Zhou X, Chen C, Dong Q, Zhang H, Zhou R, Zhao H, Chen C, Qiao S, Jiang T, Guo Y. Event-related potentials of single-digit addition, subtraction, and multiplication. Neuropsychologia 2006; 44:2500-7. 